\title{
Estructuras mentales y aprendizaje autorregulado en generación de aprendizaje significativo
}

\section{Mental structures and self-regulated learning in the generation of meaningful learning}

William Ecuador Martínez Albán wilanjose@hotmail.com

Pontificia Universidad Católica del Ecuador, Extensión Manabí, Portoviejo Ecuador https://orcid.org/0000-0001-5463-7080

Patricio Alfredo Vallejo Valdivieso pvallejo@utm.edu.ec pvallejo@pucem.edu.ec

Universidad Técnica de Manabí, Portoviejo

Pontificia Universidad Católica del Ecuador, Extensión Manabí, Portoviejo

Ecuador

https://orcid.org/0000-0003-3248-7864

María Elena Moya Martínez mmoya@pucem.edu.ec

Pontificia Universidad Católica del Ecuador, Extensión Manabí, Portoviejo

Ecuador

https://orcid.org/0000-0001-7504-6933

Recibido: 29 de septiembre de 2019

Aprobado: 30 de octubre de 2019

\begin{abstract}
RESUMEN
Se trabajó con una investigación tipo correlacional transeccional en procura de determinar la relación de las estructuras mentales en el aprendizaje significativo a partir de la aplicación de estrategias basadas desde el enfoque del aprendizaje autorregulado en estudiantes de la carrera de enfermería de la universidad Técnica de Manabí, sede Portoviejo - Ecuador. Las estructuras mentales (EM) y aprendizaje significativo desde la autorregulación (APA), presentan una correlación de Pearson de ,278 siendo ubicada
\end{abstract}


en el rango de positiva baja. El aprendizaje autorregulado promueve valores éticos sociales en el estudiante, situación que permite edificar una personalidad basada en la autogeneración de aptitudes respetuosas al otro, reconociendo que cada persona posee un valor para la consolidación del aprendizaje, mediante la puesta en marcha de competencias que posibilitan el ejercicio pedagógico en un marco de construcción social del conocimiento.

Descriptores: Neuropsicología, investigación sobre el cerebro, educación alternativa, experimento educacional.

\begin{abstract}
We worked with a translational correlational type investigation in order to determine the relationship of mental structures in meaningful learning based on the application of strategies based on the approach of self-regulated learning in nursing students of the Technical University of Manabí, Portoviejo headquarters - Ecuador. Mental structures (MS) and significant learning from self-regulation (APA), have a Pearson correlation of 278 , being located in the low positive range. Self-regulated learning promotes ethical social values in the student, a situation that allows building a personality based on the self-generation of respectful skills to the other, recognizing that each person has a value for the consolidation of learning, through the implementation of skills that enable the pedagogical exercise in a framework of social construction of knowledge.
\end{abstract}

Descriptors: Neuropsychology, brain research, alternative education, educational experiments.

\title{
INTRODUCCIÓN
}

La educación progresivamente sume cambios paradigmáticos en su accionar pedagógico, en los últimos años, se ha impulsado la vertiente del enfoque centrado en el estudiante, desde el cual han surgido diversos métodos y procedimientos entre los cuales se encuentra el aprendizaje autorregulado, siendo importante por cuanto permite activar procesos mentales en los cuales se promueve operaciones neurológicas que favorecen la sinergia sobre la adquisición del aprendizaje significativo, por lo cual es importante dado que permite consolidar un aprendizaje a lo largo de la vida, siendo importante dado que este enfoque es manejado por la UNESCO de cara al 2030, ante lo cual, Macías (2017), indica que: 
El estudiante debe ser protagonista de su propio aprendizaje y debe empoderarse y comprometerse con la actividad intelectual necesaria para asumir la construcción del conocimiento. Debe ser capaz de trabajar en equipo, aprendiendo a argumentar, a resolver problemas y a respetar las ideas de otros, pues es en la interacción en donde se construye una actitud ante el conocimiento, buscando información y comprometiéndose a la resolución de problemas reales y de su medio más cercano. (p. 1).

Es así como se da un giro educativo desde la perspectiva del docente al de estudiante como centro generador del aprendizaje, siendo necesaria aplicar estrategias que contribuyan a transcender al nuevo enfoque educativo, es así que la investigación tiene por objetivo determinar la relación de las estructuras mentales en el aprendizaje significativo a partir de la aplicación de estrategias basadas desde el enfoque del aprendizaje autorregulado en estudiantes de la carrera de enfermería de la universidad Técnica de Manabí, sede Portoviejo - Ecuador.

\section{DESARROLLO}

\section{Aprendizaje autorregulado}

El aprendizaje autorregulado promueve la autonomía, proactividad, responsabilidad, en el estudiante con la finalidad de ser el protagonista de su aprendizaje, Gaeta (2011), plantea la autorregulación del aprendizaje "implica la interrelación de procesos motivacionales y metacognitivos, como una forma de control de la acción dentro de un contexto específico" (p. 29). El aprendizaje desde lo planteado se concibe como un medio para el crecimiento e integral a lo largo de la vida, por otro lado, Zimmerman (2000), destaca que:

El aprendizaje autorregulado no es una habilidad mental, ni una destreza determinada, que se pueda aplicar en el desempeño académico. Cuando se utiliza el término de aprendizaje autorregulado, se está haciendo referencia al proceso auto-directivo (autónomo), en el cuál el alumno transforma sus habilidades mentales en destrezas relacionadas con las tareas académicas. (p.135). 
El aprendizaje autorregulado en función de lo descrito, se concibe como un proceso trasformador de competencias para la generación de un ejecutar pedagógico donde el estado pasivo del estudiante se transciende por una aptitud activa - autónoma, en posibilidad de promover el aprendizaje significativo. En complemento, Rosario, Pereira \& Hogemann, (2014) plantean que:

Los alumnos que autorregulan su aprendizaje son proactivos en cuanto a su esfuerzo por aprender, ya que son conscientes de sus habilidades y limitaciones y, además, su comportamiento de estudio está guiado por objetivos y estrategias que los ayudan a alcanzarlos. Normalmente, los alumnos que autorregulan su aprendizaje monitorizan su comportamiento en relación con sus objetivos y reflexionan sobre los avances que se van produciendo. Esta actividad promueve su satisfacción personal y su motivación para continuar y mejorar su método de aprendizaje, lo que termina repercutiendo en buenos resultados académicos y en expectativas optimistas de cara al futuro. (p. 782).

Generándose la posibilidad de estudiantes con raciocinio auto reflexivo para asumir el rol protagónico de su propio aprendizaje, siendo indispensable contar con la presencia de un docente asesor que posibilite desde la estimulación de las estructuras mentales, el aprendizaje autorregulado, Lanz (2006), plantea que "La autorregulación suele entenderse [...] como una forma de control de la acción que se caracteriza por la integración de: conocimiento metacognitivo, regulación de la cognición y motivación" ( $p$. 2).

El equilibrio racional y emocional son fundamentales en el desarrollo del aprendizaje autorregulado, implicando que el estudiante tiene la capacidad de conocer sus fortalezas y debilidades, asumiendo el compromiso consigo mismo para trabajar en ser mejor cada día, siendo un proceso auto reflexivo el responsable de contribuir en la autorregulación, Zimmerman (2000), destaca que la autorregulación es un "proceso formado por pensamientos auto-generados, emociones y acciones que están planificados y adaptadas cíclicamente para lograr la obtención de los objetivos personales" (p. 453).

El docente debe promover la conjugación de lo racional y emocional en los estudiantes, 
siendo así un aporte a consolidar personas saludables desde la educación para la conformación de una sociedad para el progreso integral, con lo cual existe una voluntad para el trabajo efectivo y de calidad en el día a día, siendo así que Woolfolk (2010), advierte que:

El aprendizaje autorregulado es la habilidad y voluntad que tiene un estudiante para analizar las tareas de aprendizaje, establecer metas y planear la forma de realizar la tarea aplicando esas habilidades y sobretodo, haciendo ajustes mientras se lleva a cabo el aprendizaje. (p.359).

Esto implica que el estudiante va regulándose en la medida que asume el aprendizaje como un desafío a superar para ser mejor persona, viéndose involucrado en procesos de resiliencia, motivación, inteligencia emocional, como factores coadyuvantes al logro de metas establecidas, viéndose reflejada la capacidad para recomponer cognitiva y emocionalmente su conducta en favor de optimizar los procesos que le permitan alcanzar un aprendizaje significativo.

\section{Fases del aprendizaje autorregulado}

El aprendizaje autorregulado desde la visión de Zimmermman (2000), plantea tres fases, las cuales son: Planificación, desempeño y reflexión, definidas así:

Durante la planificación los estudiantes establecen sus metas y estándares, los cuales deben alcanzados en una determinada tarea, sesión o curso. Esta fase involucra la percepción de los estudiantes sobre el ambiente de aprendizaje.

Durante el desempeño, los estudiantes muestran su compromiso con su experiencia de aprendizaje. Durante esta fase, los estudiantes monitorizan su aprendizaje, generalmente comparando su progreso con los estándares establecidos en la fase de planificación.

Durante la fase de reflexión, los estudiantes piensan y evalúan su experiencia de aprendizaje. Esto incluye reflexionar sobre la retroalimentación y almacenar mentalmente ideas y conceptos para usar en el aprendizaje futuro. 
Por lo tanto, el estudiante podría complementar con diversas técnicas y métodos, el desarrollo del aprendizaje autorregulado, siendo uno de ellos, lo relacionado a la estimulación de las estructuras mentales, englobándose una serie de estrategias para tal fin, por consiguiente se hace importante contribuir en diseñar estrategias didácticas desde la neurodidáctica con la finalidad de estar en concordancia con la generación de un aprendizaje autorregulado efectivo en los estudiantes, siendo indispensable para tal fin, realizar diagnósticos sobre el hemisferio cerebral dominante y estilos de aprendizaje predominante, siendo determinante para contar con una perspectiva amplia de las posibles actividades pedagógicas a desarrollar en función de establecer mayores posibilidades de consolidar el aprendizaje significativo a lo largo de la vida, posibilitándose la aparición de competencias que son adquiridas en la medida que el estudiante evoluciona y comprende la necesidad de ser protagonista de su historia de vida, por ende de su aprendizaje.

\section{Cuadro 1}

Fases del ciclo de Zimmermman

\begin{tabular}{|c|c|c|}
\hline Fases & Proceso & Descripción \\
\hline \multirow[t]{2}{*}{$\begin{array}{l}\text { Fase de } \\
\text { planificación }\end{array}$} & Análisis de la tarea & $\begin{array}{l}\text { El estudiante analiza las características de la } \\
\text { tarea, construyendo así una perspectiva de lo } \\
\text { que debe realizar }\end{array}$ \\
\hline & Automotivación & $\begin{array}{l}\text { El estudiante analiza el valor de la tarea, } \\
\text { otorgándole un valor al esfuerzo para } \\
\text { desarrollar la misma }\end{array}$ \\
\hline \multirow[t]{2}{*}{$\begin{array}{l}\text { Fase de } \\
\text { ejecución }\end{array}$} & Autoobservación & $\begin{array}{l}\text { El estudiante compara lo que está realizando } \\
\text { con el modelo a alcanzar }\end{array}$ \\
\hline & Autocontrol & $\begin{array}{l}\text { El estudiante utiliza diversas estrategias para } \\
\text { mantener la motivación y concentración }\end{array}$ \\
\hline \multirow[t]{2}{*}{$\begin{array}{l}\text { Fase de } \\
\text { autorreflexión }\end{array}$} & Auto juicio & $\begin{array}{l}\text { El estudiante juzga su accionar en la } \\
\text { actividad }\end{array}$ \\
\hline & Auto reacción & El estudiante reacciona ante los auto juicios \\
\hline
\end{tabular}

Nota: Cuadro diseñado a partir de Zimmermman (2000). 
Desde la perspectiva de Zimmermman (2000), el estudiante conjuga una serie de acciones cognitivas y emocionales en tres momentos diversos para construir un aprendizaje donde el posee el auto control de las acciones a seguir, las cuales se encuentran fundamentadas por la motivación, debido que esta influye directamente en la realización o no de las metas académicas previstas por el estudiante. Por otro lado, se plantean las fases de acuerdo a Pintrich (2000):

1. Previsión, planificación y activación: que conlleva la programación, el establecimiento de metas de aprendizaje, como así también considerar la incidencia del conocimiento personal, el contexto y los conocimientos previos sobre las tareas académicas a realizar.

2. Monitoreo: fase en la que se activa una mayor conciencia metacognitiva, para realizar un seguimiento de los aspectos personales, de la misma tarea y del contexto que repercuten sobre el rendimiento.

3. Control: en la que se llevan a cabo diversos procesos de control y de regulación sobre sí mismo, el contexto y la tarea.

4. Reflexión y reacción: en la que el sujeto se autoevalúa y evalúa el contexto y la tarea, para posteriormente reaccionar modificando algún aspecto que no favorece el desarrollo del proceso de aprendizaje.

Desde la perspectiva de Pintrich, (2000), se trabaja el aprendizaje autorregulado en cuatro fases, aunque se mantiene la esencia de Zimmermman (2000), solo que en el ciclo de Pintrich, (2000), se involucra el contexto donde se forma el estudiante, indicando que lo social tiene influencia sobre la autorregulación del estudiante, de ese modo, se hace un esbozo de Pintrich, (2000). 


\section{CIENCIAMATRIA}

Revista Interdisciplinaria de Humanidades, Educación, Ciencia y Tecnología

Año VI. Vol. VI. N¹0. Enero - Julio 2020

Hecho el depósito de ley: pp201602FA4721

ISSN-L: 2542-3029; ISSN: 2610-802X

Universidad Nacional Experimental Francisco de Miranda (UNEFM). Santa Ana de Coro. Venezuela

William Ecuador Martínez Albán; Patricio Alfredo Vallejo Valdivieso; María Elena Moya Martínez

\section{Cuadro 2}

Fases del ciclo de Pintrich

Áreas de regulación

Fases Cognición Motivación y Comportamiento Contexto afectos

1.Previsión, Establecimiento de Orientación [Planificación del [Percepciones
planificaciónmetas hacia metas. tiempo y del de la tarea].

y activación esfuerzo].

Activación del Juicios de [Planificación de [Percepciones conocimiento autoeficacia. la del contexto].

previo considerado Juicios sobre el autoobservación

relevante para la aprendizaje. del

tarea.

Activación de

conocimiento

metacognitivo.
Percepciones comportamiento].

sobre la

dificultad de la

tarea.

Activación del

valor y del

interés

personal sobre

la

tarea.

\section{MonitoreoConciencia}

metacognitiva y

monitoreo de la cognición.
Conciencia y Conciencia y Monitoreo de monitoreo de la monitoreo del los cambios motivación y esfuerzo, el uso producidos los afectos. del tiempo y de lasobre las necesidad de condiciones de ayuda. la tarea y el Autoobservación contexto. del comportamiento.

3. Control Selección y adaptación de estrategias cognitivas de aprendizaje.

$\begin{array}{lll}\text { Selección y } & \text { Aumento y } & \text { Modificar o } \\ \text { adaptación de } & \text { disminución del } & \text { renegociar } \\ \text { estrategias de } & \text { esfuerzo. } & \text { tarea. Modificar } \\ \text { gestión de la } & \text { Persistencia } & \text { o salir del } \\ \text { motivación y } & \text { Renuncia } & \text { contexto. }\end{array}$


Pensamiento. los Búsqueda de

afectos. ayuda
4. Reacción Juicios cognitivos. Reacciones
Evaluación de
y reflexión Atribuciones.
afectivas.
la tarea.
Atribuciones.
Evaluación del
contexto.

Nota: (Pintrich, 2000), tomado de Daura 2010.

El estudiante parte desde una meta planificada, proyectándose una visión que permite tener en consideración la importancia que tiene la disposición del estudiante para realizar las diversas actividades que conforman la meta a alcanzar, siendo que el aprendizaje autorregulado se gestiona como un proceso disciplinario donde se abordan los diversos principios de un aprendizaje autónomo, dinámico, flexible, donde se concibe al estudiante como centro del proceso formativo.

\section{Docente en el aprendizaje autorregulado}

El docente en el aprendizaje autorregulado juega un papel fundamental en la medida que motiva y guía al estudiante al logro del mismo, por cuanto el estudiante que proviene de un esquema instruccional de enseñanza, necesita acompañamiento para tener una transición entre un modelo mecanicista a uno donde es autónomo de construir el aprendizaje, allí el docente debe tener una visión reflexiva para sensibilizar al estudiante de la implementación de este estilo de aprendizaje, sobre todo cuando los intereses y necesidades del estudiante no se encuentren en concordancia con generar un aprendizaje a lo largo de la vida. En este sentido, Paris et. al. (2001), indica que:

Con el fin de tener éxito, los profesores deben ser reflexivos y analíticos sobre sus propias creencias y práctica, y que debe adquirir un profundo conocimiento de los principios cognitivos y de motivación de aprendizaje y enseñanza. Con este fin, se analiza como los profesores pueden modelar y promover la autorregulación del 
aprendizaje de sus alumnos. (p. 1).

Por consiguiente es un docente que debe creer en sí mismo para hacer creer a los estudiantes en ellos mismos, siendo este una particularidad que les permitirá reflexionar para lograr la articulación de un aprendizaje mediado por un equipo de trabajo conformado por docente y estudiantes, ambos investigan para conocer y alcanzar las metas propuestas, así se germina el rol asesor del docente, a juicio de Talavera de Fernández y Fernández (2018),

El concepto de asesoría que ha sido relacionado con el proceso enseñanzaaprendizaje, es intrínseco al desarrollo del individuo, quien de acuerdo a la pedagogía moderna es un ser naturalmente bueno, consciente de sus propias necesidades en proceso de evolución y con aptitudes en función de su interacción en el medio; su aprendizaje se efectúa mediante un proceso cognoscitivo y de condicionamiento operante, motivado según sus metas, necesidades, experiencia y percepción de la necesidad de resolver problemas. (p. 4).

Por lo tanto, el docente debe mirar las potencialidades de los estudiantes para incentivarlos a ser mejores personas y por ende a motivarlos al logro de un aprendizaje que les permita construir conocimientos a lo largo de la vida, de ese modo se genera una relación donde el docente con base a una visión humanista contribuye al uso de estrategias, métodos pedagógicos, que contribuyan al fortalecimiento del aprendizaje autorregulado como generador de un equilibrio en la personalidad del estudiante con la función de centrarse en el trabajo autónomo para el logro de las metas planificadas. En complemento, Zapata (2018, p. 2), plantea que las funciones del docente mediador son: Propiciar espacios de colaboración para que tanto el profesor como los estudiantes participen activamente de los procesos didácticos, trabajen en equipo, intercambien experiencias y conocimientos en una relación dialogante entre pares donde todos tienen algo que aportar.

Fomentar el desarrollo de la autonomía de los estudiantes con acciones encaminadas a descubrir métodos eficientes de estudio que les permitan aprender a aprender. 
Facilitar el aprendizaje significativo con estrategias guiadas que apunten al desarrollo de habilidades y a la solución de problemas en la vida real.

Fomentar la creatividad ofreciendo espacios para que los estudiantes enfrenten y resuelvan situaciones problema, y que se aventuren a proponer ideas originales en un ambiente de respeto por las ideas divergentes.

Incentivar el desarrollo de valores humanos como la responsabilidad y disciplina, la solidaridad, el respeto, la tolerancia, y la humildad ante el conocimiento; todo con el fin de formar sujetos útiles a la sociedad.

Desarrollar habilidades comunicativas que le permitan por un lado hacer la representación simbólica de los contenidos, y por otro, relacionarse con los estudiantes en forma cercana y afectuosa, para conocer las dificultades y demandas de cada estudiante en particular, y poder ofrecerle asesoría personalizada que genere seguridad, motivación y confianza en los estudiantes.

Promover mediante procesos de evaluación de los aprendizajes habilidades metacognitivas en el estudiante, con el fin, de que este reflexione sobre la eficacia de sus métodos de aprendizaje, para que autoevalúe sus logros, busque mejorar sus esquemas internos de comprensión de significados y atribución de sentido y, para que construya conocimientos autorregulados acorde sus capacidades y habilidades de aprendizaje.

En resumen, el docente debe concebir la educación como un proceso mediante el cual se alcanza competencia meta cognitiva en el estudiante para que este pueda ser autónomo en la construcción de su aprendizaje, situación que le permitirá contribuir en la conformación de un estudiante en capacidad de asumir retos para formarse permanente dentro y fuera de la escuela, con lo cual, se proyecta una persona critica, reflexiva, innovadora, preocupada por cultivarse en función de ser un ciudadano integral, siendo esta una de las premisas del aprendizaje a lo largo de la vida.

Metodología de la investigación

Se trabajó con una investigación tipo correlacional transeccional en procura de 


\section{CIENCIAMATRIA}

Revista Interdisciplinaria de Humanidades, Educación, Ciencia y Tecnología

Año VI. Vol. VI. N¹0. Enero - Julio 2020

Hecho el depósito de ley: pp201602FA4721

ISSN-L: 2542-3029; ISSN: 2610-802X

Universidad Nacional Experimental Francisco de Miranda (UNEFM). Santa Ana de Coro. Venezuela

William Ecuador Martínez Albán; Patricio Alfredo Vallejo Valdivieso; María Elena Moya Martínez

determinar la relación de las estructuras mentales en el aprendizaje significativo a partir de la aplicación de estrategias basadas desde el enfoque del aprendizaje autorregulado en estudiantes de la carrera de enfermería de la universidad Técnica de Manabí, sede Portoviejo - Ecuador, sustentándose metodológicamente desde lo propuesto por Hernández, Fernández y Baptista (2014).

La población objeto de estudio estuvo conformado por 19 estudiantes de la carrera de enfermería de la materia morfofisiología a quienes se les aplicó una serie de estrategias basadas en el aprendizaje autorregulado con la finalidad de estimular las estructuras mentales, situación que permitió medir la relación con el aprendizaje significativo alcanzado en la materia, siendo necesario la aplicación de un instrumento que permitió medir las variables, siendo en escalamiento de Likert de cinco alternativas, obteniéndose un valor de 0.91 para el coeficiente de Alfa de Cronbach, catalogándose como altamente confiable.

\section{RESULTADOS}

\section{Cuadro 1}

Estructuras mentales y aprendizaje significativo desde la autorregulación

\begin{tabular}{llr|r} 
& & \multicolumn{1}{c}{ EM } & \multicolumn{1}{c}{ APA } \\
\hline \multirow{2}{*}{ EM } & Correlación de Pearson & 1 &, 278 \\
\cline { 2 - 4 } & Sig. (bilateral) & &, 249 \\
\cline { 2 - 4 } & $\mathrm{N}$ & 19 & 19 \\
\hline \multirow{2}{*}{ APA } & Correlación de Pearson &, 278 & 1 \\
\cline { 2 - 4 } & Sig. (bilateral) &, 249 & \\
\cline { 2 - 4 } & $\mathrm{N}$ & 19 & 19 \\
\hline
\end{tabular}

Las estructuras mentales (EM) y aprendizaje significativo desde la autorregulación 
(APA), presentan una correlación de Pearson de ,278 siendo ubicada en el rango de positiva baja, aunque existe correlación se hace pertinente profundizar en la práctica de ambas variables con la finalidad de incrementar el grado significancia con la intención de acercarse a 1, con lo cual se incrementaría la perfección de la correlación en próximas investigaciones.

Discusión

El aprendizaje autorregulado implica la generación de diversos procesos, donde se involucra lo mental como eje fundamental para lograr la autorregulación, en ese sentido, cobra pertinencia lo planteado por lanni Gómez (2017), sobre la necesidad de generar praxis pedagógica desde una visión holística que permita involucrar las complejidades del ser con su entorno, siendo así que se promueve a partir de los resultados alcanzados, un giro en el modo de generar aprendizajes en estudiantes universitarios de la carrera enfermería a juicio de Vallejo Valdivieso, Zambrano Pincay, Vallejo Pilligua \& Bravo Cedeño (2019), esto implica la posibilidad de tener en cuenta los aspectos Biopsicosociales más efectos neuroendocrinos durante el proceso de aprendizaje con la finalidad de posibilitar el aprendizaje significativo, esto promueve el accionar hacia un modelo educativo enfocado en el estudiante como sujeto activo - protagónico, siendo indispensable contar con el respaldo de la gerencia educativa para promover el enfoque planteado con mayor pertinencia en la institución, tal como lo proponen Morgado, Aldana Zavala \& Isea Argüelles (2019).

\section{CONCLUSIONES}

El aprendizaje autorregulado fomenta el aprendizaje significativo por cuanto está concebido para generar en el estudiante la autonomía didáctica en procura de aprender investigando, lo cual requiere involucrar múltiples áreas entre lo cual, las estructuras mentales juegan un papel importante, siendo necesaria la estimulación adecuada por parte del docente con la finalidad de generar en el estudiante, la posibilidad de motivarse en aprender desde una connotación colaborativa y cooperativa, por cuanto 
no solo aprende aislado, sino, en compañía del conjunto de compañeros, aunados a las experiencias de vida, generándose sinergia que confluye en la consecución de metas grupales.

El aprendizaje autorregulado promueve valores éticos - sociales en el estudiante, situación que permite edificar una personalidad basada en la autogeneración de aptitudes respetuosas al otro, reconociendo que cada persona posee un valor para la consolidación del aprendizaje, mediante la puesta en marcha de competencias que posibilitan el ejercicio pedagógico en un marco de construcción social del conocimiento.

\section{REFERENCIAS CONSULTADAS}

1. Daura, F. (2010). El aprendizaje autorregulado y su orientación por parte del docente universitario. Recuperado de http://www.adeepra.org.ar/congresos/Congreso\%20IBEROAMERICANO/DOCEN TES/RLE2992_Daura.pdf

2. Gaeta, M.L.,Teruel, M.P. y Orejudo, S. (2011). Motivational, volitional and metacognitive aspects of self regulated learning. Electronic Journal of Research in Educational Psychology, 10(1), 73-94.

3. Hernández, R. Fernández, C., y Baptista, P. (2014). Metodología de la investigación. México, Mc Graw Hill Hispanoamericana. Hill Internacional

4. Ianni Gómez., L. (2017). Miramda: Una propuesta educativa emergente desde la investigación. Revista Arbitrada Interdisciplinaria Koinonía, 2(3), 9-30. Recuperado de http://fundacionkoinonia.com.ve/ojs/index.php/revistakoinonia/article/view/49/36

5. Lanz, M. (2006). Hacia la comprensión del aprendizaje autorregulado. Recuperado http://investigacion.ilce.edu.mx/panel_control/doc/lect_autorreg.pdf

6. Macías, M. (2017). El alumno como protagonista de su propio aprendizaje. Recuperado de https://ineverycrea.mx/comunidad/ineverycreamexico/recurso/elalumno-como-protagonista-de-su-propio/765cad1d-0696-43bb-9add$649 a 7 e 1 c 5650$

7. Morgado, C., Aldana Zavala, J., \& Isea Argüelles, J. (2019). Gerencia 
transformacional desde el accionar docente directivo para el abordaje del $\begin{array}{llll}\text { Contexto } & \text { Escolar. } & \text { CIENCIAMATRIA, } & \text { 48-72. }\end{array}$ https://doi.org/10.35381/cm.v5i9.99

8. Paris, S.G., Byrnes, J.P. y Paris, A.H. (2001). Constructing theories, identities, and actions of self-regulated learners. En B.J. Zimmerman y D.H. Schunk (Eds.), Self-regulated learning andacademic achievement: Theoretical perspectives(pp. 253-287). Hillsdale, NJ: Erlbaum.

9. Pintrich, P.R. (2000b). The role of goal orientation in self-regulated learning. En M. Boekaerts, P.R. Pintrich y M. Zeidner (Eds.), Handbook of self-regulation(pp. 451-502). San Diego, CA: Academic Press.

10. Rosário, P., Pereira, A.,Högemann, J., Nunes, A. R., Figueiredo, M., Núñez, J. C., Fuentes, S., Gaeta, M.L. (2014). Autorregulación del aprendizaje: una revisión sistemática en revistas de la base SciELO. Universitas Psychologica, 13(2), 781798. doi:10.11144/Javeriana.UPSY13-2.aars

11. Talavera de Fernández, E., y Fernández, V. (2018). El docente investigador, asesor y facilitador y su relación con la calidad en las tesis de grado de los alumnos cursantes del décimo semestre de la mención educación comercial de la escuela de educación de la universidad de Carabobo. Recuperado de http://servicio.bc.uc.edu.ve/educacion/revista/a1n18/1-18-5.pdf

12. Vallejo Valdivieso, P., Zambrano Pincay, G., Vallejo Pilligua, P., \& Bravo Cedeño, G. (2019). Estructuras mentales en la construcción de aprendizaje significativo. CIENCIAMATRIA, 5(8), 228-241. Recuperado a partir de http://cienciamatriarevista.org.ve/index.php/cm/article/view/257

\section{REFERENCES CONSULTED}

1. Daura, F. (2010). Self-regulated learning and orientation by the university teacher.

http://www.adeepra.org.ar/congresos/Congreso\%20IBEROAMERICANO/DOCEN TES/RLE2992_Daura.pdf

2. Gaeta, M.L., Teruel, M.P. and Orejudo, S. (2011). Motivational, volitional and metacognitive aspects of self regulated learning. Electronic Journal of Research in Educational Psychology, 10 (1), 73-94. 
3. Hernández, R. Fernández, C., and Baptista, P. (2014). Investigation methodology. Mexico, Mc Graw Hill Hispanic American. Hill International

4. Ianni Gómez., L. (2017). Miramda: An educational proposal emerging from research. Interdisciplinary Arbitrated Review Koinonía, 2 (3), 9-30. Recovered from

http://fundacionkoinonia.com.ve/ojs/index.php/revistakoinonia/article/view/49/36

5. Lanz, M. (2006). Towards the understanding of self-regulated learning. Recovered http://investigacion.ilce.edu.mx/panel_control/doc/lect_autorreg.pdf

6. Macías, M. (2017). The student as the protagonist of their own learning. Recovered from https://ineverycrea.mx/comunidad/ineverycreamexico/recurso/elalumno-como-protagonista-de-su-propio/765cad1d-0696-43bb-9add$649 \mathrm{a} 7 \mathrm{e} 1 \mathrm{c} 5650$

7. Morgado, C., Aldana Zavala, J., \& Isea Argüelles, J. (2019). Transformational management from the directive teaching actions to address the School Context. SCIENCE, 5 (9), 48-72. https://doi.org/10.35381/cm.v5i9.99

8. Paris, S.G., Byrnes, J.P. and Paris, A.H. (2001). Constructing theories, identities, and actions of self-regulated learners. In B.J. Zimmerman and D.H. Schunk (Eds.), Self-regulated learning andacademic achievement: Theoretical perspectives (pp. 253-287). Hillsdale, NJ: Erlbaum.

9. Pintrich, P.R. (2000b). The role of goal orientation in self-regulated learning. In M. Boekaerts, P.R. Pintrich and M. Zeidner (Eds.), Handbook of self-regulation (pp. 451-502). San Diego, CA: Academic Press.

10. Rosário, P., Pereira, A., Högemann, J., Nunes, A. R., Figueiredo, M., Núñez, J. C., Fuentes, S., Gaeta, M.L. (2014). Self-regulation of learning: a systematic review in journals of the SciELO base. Universitas Psychologica, 13 (2), 781-798. doi: 10.11144 / Javeriana.UPSY13-2.aars

11. Talavera de Fernández, E., and Fernández, V. (2018). The research teacher, advisor and facilitator and its relationship with the quality in the thesis of the students attending the tenth semester of the mention commercial education of the school of education of the University of Carabobo. Recovered from http://servicio.bc.uc.edu.ve/educacion/revista/a1n18/1-18-5.pdf 


\section{CIENCIAMATRIA}

Revista Interdisciplinaria de Humanidades, Educación, Ciencia y Tecnología

Año VI. Vol. VI. N¹0. Enero - Julio 2020

Hecho el depósito de ley: pp201602FA4721

ISSN-L: 2542-3029; ISSN: 2610-802X

Universidad Nacional Experimental Francisco de Miranda (UNEFM). Santa Ana de Coro. Venezuela

William Ecuador Martínez Albán; Patricio Alfredo Vallejo Valdivieso; María Elena Moya Martínez

12. Vallejo Valdivieso, P., Zambrano Pincay, G., Vallejo Pilligua, P., \& Bravo Cedeño, G. (2019). Mental structures in the construction of meaningful learning. SCIENCE, 5 (8), 228-241. Recovered from http://cienciamatriarevista.org.ve/index.php/cm/article/view/257

C2020 por los autores. Este artículo es de acceso abierto y distribuido según los términos y condiciones de la licencia Creative Commons Atribución-NoComercial-Compartirlgual 4.0 Internacional (CC BY-NC-SA 4.0)

(https://creativecommons.org/licenses/by-nc-sa/4.0/). 